\title{
Attitude Deference among Suicide Attempters and Non Attempters: An Indian Perspective
}

\author{
Raja lakshmi Ramui ${ }^{*}$, Vijayalakshmi Poreddi², Rohini Thimmaiah ${ }^{3}$, Sugavana Selvi ${ }^{4}$ and Suresh Bada Math ${ }^{5}$ \\ ${ }_{1,2,4}$ College of nursing, National Institute of mental health and Neuro sciences-Institute of National Importance, Bangalore, India \\ ${ }^{3}$ Toowoomba Base Hospital, Australia
}

${ }^{5}$ Department of Psychiatry, National Institute of mental health and Neuro sciences, Institute of National Importance, Bangalore, India

Submission: June 26, 2017; Published: July 31, 2017

*Corresponding author: Rajalakshmi Ramu, College of Nursing, National Institute of Mental Health and Neuro Sciences, Institute of National Importance, Bangalore 560 029, India, Tel: 9742013999; Email: radhikanmj@gmail.com

\begin{abstract}
Aim: The aim of this paper is to conduct an analysis of attitude differences in the outcome of a broad range of suicidal behavior occurring within the general publics.

Methodology: This study was a cross sectional descriptive survey carried out among general population residing in an urban community. data.

Data analysis: Descriptive (frequency and percentage) and inferential statistics (chi square test) were used to analyze and interpret the
\end{abstract}

Result: There are few significant attitude difference found between the suicide attempters and non-attempters of the general public's. Majority of the respondent believe that People who make suicidal threats seldom complete suicide, one should rather not talk about, it and suicide can happen without warning.

Conclusion: These attitude differences need to be taken in to consideration when developing appropriate programs to prevent suicide. Raising wakefulness about suicide avoidance among general population is vital in developing countries like India. Since the causes of suicides are multiple, there is no single solution that can prevent all suicides. The prevention program need to be tailored for different age, sex, cause and setting.

Keywords: Non attempters; Suicide; Attempters; Attitudes

\section{Introduction}

Suicide has become a major health care problem today. It is frequently said, "People carrying out suicides leave their skeletons behind". The loss of a young person or an attempt leaves infinite scars for the survivors and their family members, in a society where suicides are highly stigmatized. For those who attempt, the psychosocial problems are huge and live with them for rest of their life [1]. Suicide and suicide attempts are a major source of death and morbidity in global. For every accomplished suicide, there are somewhere between 10 and 40 attempted suicides [2,3].

Other sources estimate that there are 10-25 nonfatal suicide attempts for every suicide completion, and these numbers rise to 100-200 for adolescents [4]. In addition, high rates of suicide have been well documented in India [5-8]. However, recent reports from Vellore in southern India have revealed that suicide rates in India are grossly underreported.

In Karnataka, the number of people ending their life in a voluntary/deliberate act has varied from 12 to 13,000 per year during the years 2005 to 2007, with 12,304 suicides in 2007 (rate of 21.6/100,000 population) Suicides in Bangalore [1].

The general population ratio of suicide attempts to completed suicide varies with such factors as age, sex, ethnicity, co morbid conditions, and the accuracy of case recognition, particularly for suicide attempts of varied brutality and potential lethality [9]. These authors reflected that, given these caution, rates of attempted suicide in the general population are estimated to be $0.14-0.28 \%$ per year, compared with an average suicide rate of $0.014 \%$ per year, for a ratio of at least $10: 1$ and as high as $30: 1$ [10]. 
Farberow [11] and Farberow \& Shneidman [12] conducted study with veterans and found that, while no significant differences between the attempters and the threateners were observed in demographic details or case histories, marked differences were found in the mood of the attempters and threateners. The attempters showed a much more favorable psychiatric picture, with considerably less anxiety, depression, and hostility/aggression. It seems that, the attempt had provided a release from most of the pressures that had preceded their suicide attempt.

Attitudes are the key concept in the socio-psychological model of explaining and predicting human behaviour and social construction of the world around us [13]. They are defined as lasting cognitive, emotional and active predispositions towards a certain object [14].

The aim of this present study was to conduct a analysis of attitude differences in the outcome of a broad range of suicidal behavior occurring within the general public's. The objectives were identifying socio demographic characters and assessing the attitude difference between attempters and non attempters of suicidal action.

\section{Materials and Methods}

This study was a cross sectional descriptive survey carried out among general population residing in an urban community. In this study the respondents were randomly selected from the government's household registry. The sample under study was randomly selected from 1897 of 436 households in a geographically defined area of Bangalore City. The total population of this selected community is 1897, of whom $47.7 \%$ $(n=905)$ were belonged to $18-65$ years age group. Majority $(n=241,57.38 \%)$ were Muslims followed by 39.76\% ( $n=167)$ of Hindus and $2.86 \%(n=12)$ of Christians and contradicting with 2011 census that shown majority population (80.5\%) belongs to Hindus. However this area was selected considering logistic and feasibility present study was conducted. House to house survey was done among $50 \%$ of the randomly selected houses. The inclusion criteria for the present study was

a. Above 18 years of age or older.

b. Individuals who had lived in the target community for at least three months in the six months prior to the survey.

c. Who were willing to participate in the study

Persons those are suffering with severe psychiatric illnesses and cognitive disorders were excluded. A total of 216 individuals were invited to participate in the study. However, after exclusion of individuals who refused to participate including eight of the approached suicide attempters $(n=21)$ and those who could not be reached after several home visits $(n=23)$, the final sample consisted of 172 individuals.

\section{Data collection instruments}

The questionnaire has two sections

a. Personal data included information about the gender, age, marital status, family monthly income, education and religion of participants.

b. The Attitudes towards Suicide questionnaire (ATTS) was used to measure participants attitudes towards suicide and suicidal behavior [15].

The original version of tool consisted of 61 items. The present study adopted first three items to measure exposure to suicidal problems (ideation and attempts) and suicide by self and significant others in the family and outside the family [16]. For example:

a. Have you attempted suicide?

b. Is there anyone in your closest surroundings that has had or has suicidal thoughts, has expressed suicidal plans or has threatened to take their life?

i. In the family (father/mother, child, husband/wife, girlfriend/boyfriend.)

ii. Others (other relatives, friends, work- and schoolmates, others).

c. Has anyone you personally know committed suicide?

The ATTS includes 37 statements (to measure attitudes) about suicidal behavior with a five-point Likert answering scale. The attitude items present a view on suicide, for example, "People have a right to commit suicide" and the respondents were asked to give a response on a five point scale ranging from 1=Strongly disagree to $5=$ Strongly agree. The higher scores therefore represent grater agreement with the items. The reliability coefficients vary from 0.38 to 0.86 for the scale [15]. Data on internal consistency of the instrument are presented in the results part and other psychometric properties of the original ATTS questionnaire have been well documented. The ATTS is also appropriate for a wide range of populations; it is not limited for use among certain age groups, people with specific cultural backgrounds, or those working in certain professional disciplines.

\section{Data collection procedure}

The researchers themselves reached the selected houses. The family members were asked about pedigree charting and head of the family was invited to participate (women in case of absence of men) after explaining purpose of the study by the researchers and taking the written consent from the participants. English version of the questionnaire was used for this present study. Data was collected through face to face interview format at the participant's home. Despite of the random sampling procedure, individuals without education and primary education and 
women were substantially overrepresented in our sample could be due to data was collected during working hours.

\section{Ethical considerations}

The study protocol was reviewed and approved by the Ethics Committee of the Dr. BR Ambedkar Medical College, Bangalore. The aims and purpose of the study were thoroughly explained to all participants and written informed consent was taken. Participation was voluntary, their information was kept confidential and those in need were referred for psychiatric consultation.

\section{Result}

Table 1: Association of Socio-demographic detail of study subjects and Suicide attempt status (n=172).

\begin{tabular}{|c|c|c|c|c|c|c|c|}
\hline \multirow{3}{*}{ Variables } & \multirow{3}{*}{ Groups } & \multicolumn{2}{|c|}{ Attempts Status } & \multirow[t]{2}{*}{ Total } & \multirow[t]{2}{*}{$\mathbf{X}^{2}$} & \multirow[t]{2}{*}{ Df } & \multirow[t]{2}{*}{ P-Value } \\
\hline & & Yes & No & & & & \\
\hline & & $n(\%)$ & $n(\%)$ & $n(\%)$ & \multirow{6}{*}{$1.843^{\mathrm{a}}$} & \multirow{6}{*}{4} & \multirow{6}{*}{.765} \\
\hline \multirow[t]{5}{*}{ Age } & $<25$ & $8(27.6)$ & $37(29.6)$ & $45(26.2)$ & & & \\
\hline & $26-35$ & $11(37.9)$ & $50(35.0)$ & $61(35.5)$ & & & \\
\hline & $36-45$ & $6(20.7)$ & $34(23.8)$ & $40(23.3)$ & & & \\
\hline & $46-55$ & $4(13.8)$ & $15(10.5)$ & $19(11.0)$ & & & \\
\hline & $>55$ & $0(0)$ & $7(4.9)$ & $7(4.1)$ & & & \\
\hline \multirow[t]{3}{*}{ Marital Status } & Married & $23(79.3 \%)$ & $119(83.2 \%)$ & $142(82.6 \%)$ & \multirow{3}{*}{$0.338^{\mathrm{a}}$} & \multirow{3}{*}{2} & \multirow{3}{*}{.845} \\
\hline & Unmarried & $5(17.2 \%)$ & $21(14.7 \%)$ & $26(15.1 \%)$ & & & \\
\hline & $\begin{array}{l}\text { Widowed/ } \\
\text { Separated }\end{array}$ & $1(3.4 \%)$ & $3(2.1 \%)$ & $4(2.3 \%)$ & & & \\
\hline \multirow[t]{2}{*}{ Gender } & Male & $12(41.4 \%)$ & $44(30.8 \%)$ & $56(32.6 \%)$ & \multirow{2}{*}{$1.236^{\mathrm{a}}$} & \multirow{2}{*}{1} & \multirow{2}{*}{.266} \\
\hline & Female & $17(58.6 \%)$ & $99(69.2 \%)$ & $116(67.4 \%)$ & & & \\
\hline \multirow[t]{3}{*}{ Religion } & Hindu & $12(41.4 \%)$ & $56(39.2 \%)$ & $68(39.5 \%)$ & \multirow{3}{*}{$.671^{\mathrm{a}}$} & \multirow{3}{*}{2} & \multirow{3}{*}{.715} \\
\hline & Muslim & $16(55.2 \%)$ & $76(53.1 \%)$ & $92(53.5 \%)$ & & & \\
\hline & Christian & $1(3.4 \%)$ & $11(7.7 \%)$ & $12(7.0 \%)$ & & & \\
\hline \multirow[t]{4}{*}{ Education } & Illiterate & $7(24.1 \%)$ & $40(28.0 \%)$ & $47(27.3 \%)$ & \multirow{4}{*}{$1.976^{\mathrm{a}}$} & & \multirow{4}{*}{.577} \\
\hline & Primary & $15(51.7 \%)$ & $56(39.2 \%)$ & $71(41.3 \%)$ & & & \\
\hline & Secondary & $6(20.7 \%)$ & $35(24.5 \%)$ & $41(23.8 \%)$ & & & \\
\hline & Graduation & $1(3.4 \%)$ & $12(8.4 \%)$ & $13(7.6 \%)$ & & & \\
\hline
\end{tabular}

The Table 1 revealed that majority of the study subjects those who attempted for suicide $\mathrm{n}=11(37.9 \%)$ and not attempted for suicide $n=50(35.0 \%)$ were from the age group of 26-35 years, also there is no significant association between their attitudes towards suicide (X2=0.843, $\mathrm{P}=0.765)$. Majority of the subjects those who attempted for the suicide $n=23(79.3 \%)$, not attempted $=119(83.2 \%)$ were from married category, there is no significant association between married, unmarried and other category $(\mathrm{X} 2=0.338 \mathrm{a}, \mathrm{P}=0.845)$. Majority of the study subjects were females that is attempted for the suicide $\mathrm{n}=17(58.6 \%)$, not attempted for the suicide were, $\mathrm{n}=99(69.2 \%)$ and there is no significant association between attitudes of the male and female study subjects $(X 2=1.236, \mathrm{P}=0.266)$. Majority of the study subjects those who attempted $n=16(55.2 \%)$ and not attempted for the suicide $n=76(53.1 \%)$ were from Muslim category, also there is no significant association between the

\section{Statistical analysis}

The data were analyzed using appropriate statistical procedures and the results were presented in narratives and tables. Descriptive (frequency and percentage) and inferential statistics (chi square test) were used to interpret the data. Wherever numbers were less in a category, those categories were clubbed while doing chi-square analysis. Prevalence of self reported suicidal expression was classified as an affirmative response to any of the questions, i.e. all response alternatives except "never" were aggregated. The results were considered significant at $\mathrm{p}<0.05$.

attitudes of the study subjects based on the religion $(X 2=0.671$, $\mathrm{P}=0.577)$. Majority of the study subjects those who attempted $\mathrm{n}=15(51.7 \%)$ and not attempted for the suicide, $\mathrm{n}=56(39.2 \%)$ were from Muslim category, also there is no significant association between the attitudes of the study subjects based on the religion $(\mathrm{X} 2=0.671, \mathrm{P}=0.577)$. Majority of the respondents who attended, $\mathrm{n}=15(51.7 \%)$ and not attended for the suicide, $\mathrm{n}=56(39.2 \%)$ were studied primary education, there is no significant association between them based on the education (X2=1.976, P-Value=0.577).

Based on the Table 2, majority, $n=26(89.6 \%)$ of the attempted and $109(76.3 \%)$ of the non attempted for suicide expressed and agreed that it is always possible to help a person having suicidal thoughts, there is no significant association between them was found through Chi Square test. Only 24(82.2\%) of the attempted and $112(77.5 \%)$ of the non attempted respondents 
expressed and agreed that suicide can never be justified. Only $8(27.6 \%)$ of the respondents those who attempted for the suicide and $48(33.6 \%)$ of the non attempted for suicide agreed and strongly said that people who commit suicide are usually mentally ill there is no significant association between them also. Only $24(82.7 \%)$ of the attempted and $111(77.7 \%)$ of the non attempted study respondents agreed and strongly said that it is human duty to try to stop someone from committing suicide. Again only $10(34.5 \%)$ of the study respondents those who attempted for the suicide and $46(39.2 \%)$ of the non attempted expressed, agreed and strongly said that they would consider the possibility of taking their life if they suffer from a sever incurable disease . seven $(24.1 \%)$ of the attempted and $49(34.3 \%)$ of the non attempted agreed and strongly said that people who make suicidal threats seldom complete suicide and there is significant association between them also(X2=9.544,P=0.049*). Similarly another $11(37.9 \%)$ of the attempted and $49(34.3 \%)$ of the non attempted study respondents agreed and strongly said that Suicide is a subject that one should rather not talk about, it showed significant association between them also (X2=10.327, $\mathrm{P}=0.035^{*}$ ). The study respondents, those who attempted for the suicide, $n=15(51.7 \%)$ and $94(65.8 \%)$ of the non attempted expressed, agreed and strongly said that suicide happen without warning and there is significant association between them were present $\left(\mathrm{X} 2=13.274, \mathrm{P}=0.010^{*}\right)$.

Table 2: Attitude difference between attempters and non attempters of suicidal action $(n=172)$.

\begin{tabular}{|c|c|c|c|c|c|c|c|c|}
\hline \multirow{2}{*}{ Variables } & Category & SA & A & UD & DA & SDA & \multirow{2}{*}{$\mathbf{X}^{2}$} & \multirow{2}{*}{ P-Value } \\
\hline & & $f(\%)$ & $f(\%)$ & $f(\%)$ & $f(\%)$ & $f(\%)$ & & \\
\hline \multirow{2}{*}{$\begin{array}{l}\text { It is always } \\
\text { possible to } \\
\text { help a } \\
\text { Person having } \\
\text { suicidal } \\
\text { thoughts }\end{array}$} & Attempters & $13(44.8)$ & $13(44.8)$ & $2(6.9)$ & $1(3.4)$ & 0 & & \\
\hline & $\begin{array}{c}\text { Non- } \\
\text { Attempters }\end{array}$ & $50(35.0)$ & $59(41.3)$ & $12(8.4)$ & $19(13.3)$ & $3(2.1)$ & 3.395 & 0.494 \\
\hline \multirow{2}{*}{$\begin{array}{l}\text { Suicide can } \\
\text { never be } \\
\text { justified }\end{array}$} & Attempters & $8(27.6)$ & $16(55.2)$ & $3(10.3)$ & $1(3.4)$ & $1(3.4)$ & 3.159 & 0.676 \\
\hline & $\begin{array}{c}\text { Non- } \\
\text { Attempters }\end{array}$ & $56(39.2)$ & $55(38.5)$ & $22(15.4)$ & $4(2.8)$ & $5(3.5)$ & & \\
\hline $\begin{array}{l}\text { Committing } \\
\text { suicide is } \\
\text { among the } \\
\text { worst }\end{array}$ & Attempters & $15(51.7)$ & $9(31.0)$ & $5(17.2)$ & 0 & 0 & 2.366 & 0.5 \\
\hline $\begin{array}{l}\text { thing to do to } \\
\text { ones relatives }\end{array}$ & $\begin{array}{c}\text { Non- } \\
\text { Attempters }\end{array}$ & $67(46.9)$ & $57(39.9$ & $15(10.5)$ & $4(2.8)$ & 0 & & \\
\hline $\begin{array}{l}\text { Most suicide } \\
\text { attempts are }\end{array}$ & Attempters & $6(20.7 \%)$ & $13(44.8)$ & $6(20.7)$ & $3(10.3)$ & $1(3.4)$ & 7.378 & 0.117 \\
\hline $\begin{array}{l}\text { impulsive } \\
\text { actions }\end{array}$ & $\begin{array}{c}\text { Non- } \\
\text { Attempters }\end{array}$ & $27(18.9)$ & $87(60.8)$ & $18(12.6$ & $11(7.7)$ & 0 & & \\
\hline \multirow{2}{*}{$\begin{array}{c}\text { Suicide is an } \\
\text { acceptable } \\
\text { means to } \\
\text { terminate } \\
\text { an incurable } \\
\text { disease }\end{array}$} & Attempters & $1(3.4)$ & $3(10.3)$ & $8(27.6)$ & $12(41.4)$ & $5(17.2)$ & 4.506 & 0.342 \\
\hline & $\begin{array}{c}\text { Non- } \\
\text { Attempters }\end{array}$ & $2(1.4)$ & $25(17.5)$ & $36(25.2)$ & $38(26.6)$ & $42(29.4)$ & & \\
\hline \multirow{2}{*}{$\begin{array}{l}\text { Once a person } \\
\text { has made } \\
\text { up his/her } \\
\text { mind about } \\
\text { committing } \\
\text { suicide no } \\
\text { one can stop } \\
\text { him/her }\end{array}$} & Attempters & $2(6.9)$ & $5(17.2)$ & $15(51.7)$ & $2(6.9)$ & $5(17.2)$ & 13.872 & 0.008 \\
\hline & $\begin{array}{c}\text { Non- } \\
\text { Attempters }\end{array}$ & $4(2.8)$ & $30(21.0)$ & $34(23.8)$ & $48(33.6)$ & $27(18.9)$ & & \\
\hline $\begin{array}{l}\text { Many suicide } \\
\text { attempts are } \\
\text { made }\end{array}$ & Attempters & $2(6.9)$ & $13(44.8)$ & $6(20.7)$ & $5(17.2)$ & $3(10.3)$ & 3.826 & 0.43 \\
\hline $\begin{array}{l}\text { because of } \\
\text { revenge or } \\
\text { to punish } \\
\text { someone else }\end{array}$ & $\begin{array}{c}\text { Non- } \\
\text { Attempters }\end{array}$ & $27(18.9)$ & $44(30.8)$ & $29(20.3)$ & $31(21.7)$ & $12(8.4)$ & & \\
\hline
\end{tabular}


Global Journal of Intellectual \& Developmental Disabilities

\begin{tabular}{|c|c|c|c|c|c|c|c|c|}
\hline $\begin{array}{l}\text { People who } \\
\text { commit } \\
\text { suicide }\end{array}$ & Attempters & $0(0)$ & $8(27.6)$ & $7(24.1)$ & $9(31.0)$ & $5(17.2)$ & 3.581 & 0.466 \\
\hline $\begin{array}{l}\text { are usually } \\
\text { mentally ill }\end{array}$ & $\begin{array}{c}\text { Non- } \\
\text { Attempters }\end{array}$ & $14(9.8)$ & $34(23.8)$ & $39(27.3)$ & $35(25.5)$ & $21(14.7)$ & & \\
\hline \multirow{2}{*}{$\begin{array}{l}\text { It is a human } \\
\text { duty to try to } \\
\text { stop } \\
\text { someone } \\
\text { from } \\
\text { committing } \\
\text { suicide }\end{array}$} & Attempters & $15(51.7)$ & $9(31.0)$ & $3(10.3)$ & $2(6.9)$ & $0(0)$ & 2.953 & 0.566 \\
\hline & $\begin{array}{c}\text { Non- } \\
\text { Attempters }\end{array}$ & $53(37.1)$ & $58(40.6)$ & $15(10.5)$ & $12(8.4)$ & $5(3.5)$ & & \\
\hline \multirow{2}{*}{$\begin{array}{l}\text { When a } \\
\text { person } \\
\text { commits } \\
\text { suicide, it is } \\
\text { something } \\
\text { that he/she } \\
\text { has } \\
\text { considered } \\
\text { for a long } \\
\text { time }\end{array}$} & Attempters & $2(6.9)$ & $10(34.5)$ & $12(41.4$ & $5(17.2)$ & $0(0)$ & 3.598 & 0.463 \\
\hline & $\begin{array}{c}\text { Non- } \\
\text { Attempters }\end{array}$ & $15(10.5)$ & $44(30.8)$ & $44(30.8)$ & $29(20.3)$ & $11(7.7)$ & & \\
\hline \multirow{3}{*}{$\begin{array}{l}\text { There is a risk } \\
\text { of evoking } \\
\text { suicidal } \\
\text { thoughts in a } \\
\text { person's } \\
\text { mind if you } \\
\text { ask about it }\end{array}$} & & & & & & & & 0.14 \\
\hline & Attempters & $2(6.9)$ & $19(65.5)$ & $3(10.3)$ & $5(17.2)$ & 0 & 6.931 & \\
\hline & $\begin{array}{l}\text { Non- } \\
\text { ttempters }\end{array}$ & $12(8.4$ & $57(39.9$ & $32(22.4)$ & $39(27.3)$ & $3(2.1)$ & & \\
\hline \multirow{2}{*}{$\begin{array}{c}\text { People who } \\
\text { make suicidal } \\
\text { threats } \\
\text { seldom } \\
\text { complete } \\
\text { suicide }\end{array}$} & Attempters & $0(0)$ & $7(24.1)$ & $13(44.8)$ & $6(20.7)$ & $3(10.3)$ & 9.544 & $0.049 *$ \\
\hline & $\begin{array}{l}\text { Non- } \\
\text { ttempters }\end{array}$ & $9(6.3)$ & $40(28.0)$ & $29(20.3)$ & $51(35.7)$ & $14(9.8)$ & & \\
\hline \multirow{2}{*}{$\begin{array}{l}\text { Suicide is a } \\
\text { subject that } \\
\text { one should } \\
\text { rather not } \\
\text { talk about }\end{array}$} & Attempters & $2(6.9)$ & $9(31.0)$ & $6(20.7)$ & $5(17.2)$ & $7(24.1)$ & 10.327 & $0.035^{*}$ \\
\hline & $\begin{array}{l}\text { Non- } \\
\text { ttempters }\end{array}$ & $11(7.7)$ & $38(26.6)$ & $30(21.0)$ & $54(37.8)$ & $10(7.0)$ & & \\
\hline $\begin{array}{l}\text { Loneliness } \\
\text { could for me } \\
\text { be a }\end{array}$ & Attempters & $4(13.8)$ & $15(51.7$ & $3(10.3)$ & $6(20.7)$ & $1(3.4)$ & 3.087 & 0.543 \\
\hline $\begin{array}{l}\text { reason to take } \\
\text { my life }\end{array}$ & $\begin{array}{l}\text { Non- } \\
\text { ttempters }\end{array}$ & $19(13.3)$ & $56(39.2)$ & $27(18.9)$ & $27(18$. & $14(9.8)$ & & \\
\hline \multirow{2}{*}{$\begin{array}{l}\text { Almost } \\
\text { everyone has } \\
\text { at one } \\
\text { time or } \\
\text { another } \\
\text { thought about } \\
\text { suicide }\end{array}$} & Attempters & $3(10.3)$ & $16(55.2)$ & $6(20.7)$ & $2(6.9)$ & $2(6.9)$ & 5.871 & 0.209 \\
\hline & Non- & $18(12.6)$ & $56(39.2)$ & $32(22.4)$ & $33(23.1)$ & $4(2.8)$ & & \\
\hline
\end{tabular}


Global Journal of Intellectual \& Developmental Disabilities

\begin{tabular}{|c|c|c|c|c|c|c|c|c|}
\hline \multirow{2}{*}{$\begin{array}{c}\text { There may } \\
\text { be situations } \\
\text { where } \\
\text { the only } \\
\text { reasonable } \\
\text { resolution is } \\
\text { suicide }\end{array}$} & Attempters & $5(17.2)$ & $11(37.9)$ & $3(10.3)$ & $7(24.1)$ & $3(10.3)$ & 6.995 & 0.136 \\
\hline & $\begin{array}{l}\text { Non- } \\
\text { ttempters }\end{array}$ & $10(7.0)$ & $35(24.5)$ & $32(22.4)$ & $46(32.2)$ & $20(14.0)$ & & \\
\hline \multirow[b]{2}{*}{$\begin{array}{l}\text { I could say } \\
\text { that I would } \\
\text { take } \\
\text { my life } \\
\text { without } \\
\text { actually } \\
\text { meaning to } \\
\text { do so }\end{array}$} & Attempters & 0 & $7(24.1)$ & $16(55.2)$ & $4(13.8)$ & $2(6.9)$ & 8.46 & 0.076 \\
\hline & $\begin{array}{c}\text { Non- } \\
\text { ttempters }\end{array}$ & $13(9.1)$ & $18(12.6)$ & $62(43.4)$ & $44(30.8)$ & $6(4.2)$ & & \\
\hline \multirow{2}{*}{$\begin{array}{c}\text { Suicide can } \\
\text { some times } \\
\text { be a relief } \\
\text { for those } \\
\text { involved }\end{array}$} & Attempters & $2(6.9)$ & $5(17.2)$ & $7(24.1)$ & $8(27.6)$ & $7(24.1)$ & 6.929 & 0.14 \\
\hline & $\begin{array}{c}\text { Non- } \\
\text { ttempters }\end{array}$ & $16(11.2)$ & $29(20.3)$ & $30(21.0)$ & $56(39.2)$ & $12(8.4)$ & & \\
\hline \multirow[b]{2}{*}{$\begin{array}{c}\text { Suicides } \\
\text { among young } \\
\text { people are } \\
\text { particularly } \\
\text { puzzling since } \\
\text { they have } \\
\text { everything to } \\
\text { live for }\end{array}$} & Attempters & $4(13.8)$ & $6(20.7)$ & $13(44.8)$ & $4(13.8)$ & $2(6.9)$ & 6.437 & 0.169 \\
\hline & $\begin{array}{l}\text { Non- } \\
\text { ttempters }\end{array}$ & $20(14.0)$ & $42(29.4)$ & $44(30.8)$ & $35(24.5)$ & $2(1.4)$ & & \\
\hline \multirow[b]{2}{*}{$\begin{array}{l}\text { I would } \\
\text { consider the } \\
\text { possibility of } \\
\text { taking my life } \\
\text { if I were to } \\
\text { suffer from } \\
\text { a severe, } \\
\text { incurable, } \\
\text { disease }\end{array}$} & Attempters & $2(6.9)$ & $8(27.6)$ & $8(27.6)$ & $10(34.5)$ & $1(3.40$ & 5.29 & 0.259 \\
\hline & $\begin{array}{c}\text { Non- } \\
\text { ttempters }\end{array}$ & $23(16.1)$ & $25(23.1)$ & $33(23.1)$ & $42(29.4)$ & $20(14.0)$ & & \\
\hline \multirow{2}{*}{$\begin{array}{l}\text { A person } \\
\text { once they } \\
\text { have suicidal } \\
\text { thoughts } \\
\text { will never let } \\
\text { them go. }\end{array}$} & Attempters & $6(20.7)$ & $11(37.9)$ & $4(13.8)$ & $7(24.1)$ & $1(3.4)$ & 1.002 & 0.91 \\
\hline & $\begin{array}{c}\text { Non- } \\
\text { ttempters }\end{array}$ & $25(17.50$ & $50(35.0)$ & $30(21.0)$ & $31(21.7)$ & $7(4.9)$ & & \\
\hline \multirow{2}{*}{$\begin{array}{c}\text { Suicide } \\
\text { happens } \\
\text { without } \\
\text { warning. }\end{array}$} & Attempters & $2(6.9)$ & $13(44.8)$ & $3(10.3)$ & $9(31.0)$ & $2(6.9)$ & 13.274 & $.010^{*}$ \\
\hline & $\begin{array}{c}\text { Non- } \\
\text { ttempters }\end{array}$ & $29(20.3)$ & $65(45.5)$ & $30(21.0)$ & $13(9.1)$ & $6(4.2)$ & & \\
\hline \multirow{2}{*}{$\begin{array}{l}\text { Most people } \\
\text { avoid talking } \\
\text { about suicide }\end{array}$} & Attempters & $7(24.1)$ & $8(27.6)$ & $7(21.1)$ & $3(10.3)$ & $4(13.8)$ & 7.39 & 0.117 \\
\hline & $\begin{array}{c}\text { Non- } \\
\text { ttempters }\end{array}$ & $33(23.1)$ & $73(51.0)$ & $19(13.3)$ & $10(7.0)$ & $8(5.6)$ & & \\
\hline \multirow[b]{2}{*}{$\begin{array}{l}\text { If someone } \\
\text { wants to } \\
\text { commit } \\
\text { suicide, it is } \\
\text { his or her } \\
\text { business and } \\
\text { we should not } \\
\text { interfere }\end{array}$} & Attempters & $1(3.4)$ & $7(24.1)$ & $5(17.2)$ & $10(34.5)$ & $6(20.7)$ & 3.788 & 0.435 \\
\hline & $\begin{array}{c}\text { Non- } \\
\text { ttempters }\end{array}$ & $15(10.50$ & $43(30.1)$ & $20(14.0)$ & $30(21.0)$ & $35(24.5)$ & & \\
\hline
\end{tabular}


Global Journal of Intellectual \& Developmental Disabilities

\begin{tabular}{|c|c|c|c|c|c|c|c|c|}
\hline \multirow{2}{*}{$\begin{array}{l}\text { It is mainly } \\
\text { loneliness } \\
\text { that drives } \\
\text { people to } \\
\text { suicide }\end{array}$} & Attempters & $6(20.7)$ & $10(34.5)$ & $4(13.80$ & $5(17.2)$ & $4(13.8)$ & 7.531 & 0.11 \\
\hline & $\begin{array}{c}\text { Non- } \\
\text { ttempters }\end{array}$ & $45(31.5)$ & $47(32.9)$ & $31(21.7)$ & $15(10.5)$ & $5(3.5)$ & & \\
\hline \multirow{2}{*}{$\begin{array}{l}\text { A suicide } \\
\text { attempt is } \\
\text { essentially } \\
\text { a cry for help }\end{array}$} & Attempters & $4(13.8)$ & $15(51.7)$ & $10(34.5)$ & $0(0)$ & $0(0)$ & 8.604 & 0.072 \\
\hline & $\begin{array}{l}\text { Non- } \\
\text { ttempters }\end{array}$ & $33(23.1)$ & $61(42.7)$ & $27(18.9)$ & $14(9.8$ & $8(5.6)$ & & \\
\hline \multirow{2}{*}{$\begin{array}{l}\text { On the whole, } \\
\text { I do not } \\
\text { understand } \\
\text { how people } \\
\text { can take their } \\
\text { lives }\end{array}$} & Attempters & $3(10.3)$ & $13(44.8)$ & $12(41.4)$ & $1(3.4)$ & $0(0)$ & 5.014 & 0.286 \\
\hline & $\begin{array}{c}\text { Non- } \\
\text { ttempters }\end{array}$ & $18(12.6)$ & $74(51.7)$ & $34(23.8)$ & $10(7.0)$ & $7(4.9)$ & & \\
\hline \multirow{2}{*}{$\begin{array}{c}\text { Usually } \\
\text { relatives have } \\
\text { no idea about } \\
\text { what is going } \\
\text { on when a } \\
\text { person is } \\
\text { thinking of } \\
\text { suicide }\end{array}$} & Attempters & $2(6.9)$ & $15(51.7)$ & $7(24.1)$ & $3(10.3)$ & $2(6.9)$ & 3.151 & 0.533 \\
\hline & $\begin{array}{c}\text { Non- } \\
\text { ttempters }\end{array}$ & $24(16.80$ & $76(53.1)$ & $27(18.9)$ & $7(4.9)$ & $9(6.3)$ & & \\
\hline \multirow[b]{2}{*}{$\begin{array}{c}\text { A person } \\
\text { suffering } \\
\text { from } \\
\text { a severe, } \\
\text { incurable, } \\
\text { disease } \\
\text { expressing } \\
\text { wishes to die } \\
\text { should } \\
\text { get help to } \\
\text { do so }\end{array}$} & Attempters & $2(6.9)$ & $16(55.2)$ & $4(13.8)$ & $3(10.3)$ & $4(13.8)$ & 4.027 & 0.402 \\
\hline & $\begin{array}{c}\text { Non- } \\
\text { ttempters }\end{array}$ & $17(11.9)$ & $52(36.4)$ & $21(14.7)$ & $18(12.6)$ & $35(24.5)$ & & \\
\hline \multirow{2}{*}{$\begin{array}{l}\text { I am prepared } \\
\text { to help a } \\
\text { person in a } \\
\text { suicidal crisis } \\
\text { by making } \\
\text { contact }\end{array}$} & Attempters & $5(17.2)$ & $9(31.0)$ & $9(31.0)$ & $3(10.30)$ & $3(10.3)$ & 2.028 & 0.731 \\
\hline & $\begin{array}{c}\text { Non- } \\
\text { ttempters }\end{array}$ & $19(13.3)$ & $61(42.7)$ & $31(21.7)$ & $17(11.9)$ & $15(10.5)$ & & \\
\hline \multirow{2}{*}{$\begin{array}{l}\text { Anybody } \\
\text { can commit } \\
\text { suicide }\end{array}$} & Attempters & $5(17.2)$ & $13(44.8)$ & $3(10.30$ & $5(17.2)$ & $3(10.3)$ & 1.564 & 0.815 \\
\hline & $\begin{array}{c}\text { Non- } \\
\text { ttempters }\end{array}$ & $21(14.7)$ & $64(44.8)$ & $24(16.8)$ & $16(11.2)$ & $18(12.6)$ & & \\
\hline \multirow{2}{*}{$\begin{array}{c}\text { I can } \\
\text { understand } \\
\text { that people } \\
\text { suffering } \\
\text { from a severe, } \\
\text { incurable, } \\
\text { disease } \\
\text { commit } \\
\text { suicide }\end{array}$} & Attempters & $4(13.8)$ & $8(27.6)$ & $7(24.1)$ & $7(24.1)$ & $3(10.3)$ & 3.725 & 0.444 \\
\hline & $\begin{array}{c}\text { Non- } \\
\text { ttempters }\end{array}$ & $8(5.6)$ & $53(37.1)$ & $25(17.5)$ & $38(26.6)$ & $19(13.3)$ & & \\
\hline \multirow{2}{*}{$\begin{array}{l}\text { People who } \\
\text { talk about } \\
\text { suicide do } \\
\text { not commit } \\
\text { suicide }\end{array}$} & Attempters & $2(6.9)$ & $5(17.2)$ & $14(48.3)$ & $4(13.8)$ & $4(13.8)$ & 4.567 & 0.335 \\
\hline & $\begin{array}{c}\text { Non- } \\
\text { ttempters }\end{array}$ & $10(7.0)$ & $37(25.9)$ & $45(31.5)$ & $37(25.9)$ & $14(9.8)$ & & \\
\hline
\end{tabular}


Global Journal of Intellectual \& Developmental Disabilities

\begin{tabular}{|c|c|c|c|c|c|c|c|c|}
\hline \multirow{2}{*}{$\begin{array}{l}\text { People do } \\
\text { have the right } \\
\text { to take their } \\
\text { own lives }\end{array}$} & Attempters & $4(13.8)$ & $12(41.4)$ & $6(20.7)$ & $2(6.9)$ & $5(17.2)$ & 7.862 & 0.097 \\
\hline & $\begin{array}{c}\text { Non- } \\
\text { ttempters }\end{array}$ & $14(9.8)$ & $36(25.2)$ & $18(12.6)$ & $25(17.5)$ & $50(30.0)$ & & \\
\hline \multirow{2}{*}{$\begin{array}{l}\text { Most suicide } \\
\text { attempts } \\
\text { are caused } \\
\text { by conflicts } \\
\text { with a close } \\
\text { person }\end{array}$} & Attempters & $7(24.1)$ & $15(51.7)$ & $1(3.4)$ & $4(13.8)$ & $2(6.9)$ & 2.562 & 0.634 \\
\hline & $\begin{array}{c}\text { Non- } \\
\text { ttempters }\end{array}$ & $26(18.2)$ & $78(54.5)$ & $17(11.9)$ & $14(9.8)$ & $8(5.6)$ & & \\
\hline \multirow[b]{2}{*}{$\begin{array}{l}\text { I would like } \\
\text { to get help } \\
\text { to commit } \\
\text { suicide if I } \\
\text { were to suffer } \\
\text { from a severe, } \\
\text { incurable } \\
\text { disease }\end{array}$} & Attempters & $3(10.3)$ & $10(34.5)$ & $4(13.8)$ & $6(20.7)$ & $6(20.7)$ & 0.843 & 0.933 \\
\hline & $\begin{array}{c}\text { Non- } \\
\text { ttempters }\end{array}$ & $9(6.3)$ & $48(33.6)$ & $25(17.5)$ & $28(19.6)$ & $33(23.1)$ & & \\
\hline \multirow{2}{*}{$\begin{array}{c}\text { Suicide can } \\
\text { be prevented }\end{array}$} & Attempters & $7(24.1)$ & $13(44.80$ & $3(10.3)$ & $2(6.9)$ & $4(13.8)$ & 22.765 & $0.000^{*}$ \\
\hline & $\begin{array}{l}\text { Non- } \\
\text { ttempters }\end{array}$ & $50(35.0)$ & 71(49.7) & $19(13.3)$ & $3(2.1)$ & $0(0)$ & & \\
\hline \multirow{2}{*}{$\begin{array}{c}\text { It is always } \\
\text { possible to } \\
\text { help a } \\
\text { Person having } \\
\text { suicidal } \\
\text { thoughts }\end{array}$} & Attempters & $13(44.8)$ & $13(44.8)$ & $2(6.9)$ & $1(3.4)$ & 0 & 3.395 & 0.494 \\
\hline & $\begin{array}{c}\text { Non- } \\
\text { ttempters }\end{array}$ & $50(35.0)$ & $59(41.3)$ & $12(8.4)$ & $19(13.3)$ & $3(2.1)$ & & \\
\hline \multirow{2}{*}{$\begin{array}{l}\text { Suicide can } \\
\text { never be } \\
\text { justified }\end{array}$} & Attempters & $8(27.6)$ & $16(55.2)$ & $3(10.3)$ & $1(3.4)$ & $1(3.4)$ & 3.159 & 0.676 \\
\hline & $\begin{array}{c}\text { Non- } \\
\text { ttempters }\end{array}$ & $56(39.2)$ & $55(38.5)$ & $22(15.4)$ & $4(2.8)$ & $5(3.5)$ & & \\
\hline \multirow[t]{2}{*}{$\begin{array}{l}\text { Committing } \\
\text { suicide is } \\
\text { among the } \\
\text { worst thing } \\
\text { to do to ones } \\
\text { relatives }\end{array}$} & Attempters & $15(51.7)$ & $9(31.0)$ & $5(17.2$ & 0 & 0 & 2.366 & 0.5 \\
\hline & $\begin{array}{c}\text { Non- } \\
\text { Attempters }\end{array}$ & $67(46.9)$ & $57(39.9$ & $15(10.5)$ & $4(2.8)$ & 0 & & \\
\hline \multirow{2}{*}{$\begin{array}{l}\text { Most suicide } \\
\text { attempts are } \\
\text { impulsive } \\
\text { actions. }\end{array}$} & Attempters & $6(20.7 \%)$ & $13(44.8)$ & $6(20.7)$ & $3(10.3)$ & $1(3.4)$ & 7.378 & 0.117 \\
\hline & $\begin{array}{c}\text { Non- } \\
\text { Attempters }\end{array}$ & $27(18.9)$ & $87(60.8)$ & $18(12.6$ & $11(7.7)$ & 0 & & \\
\hline \multirow{2}{*}{$\begin{array}{l}\text { Suicide is an } \\
\text { acceptable } \\
\text { means to } \\
\text { terminate } \\
\text { an incurable } \\
\text { disease. }\end{array}$} & Attempters & $1(3.4)$ & $3(10.3)$ & $8(27.6)$ & $12(41.4)$ & $5(17.2)$ & 4.506 & 0.342 \\
\hline & $\begin{array}{c}\text { Non- } \\
\text { Attempters }\end{array}$ & $2(1.4)$ & $25(17.5)$ & $36(25.2)$ & $38(26.6)$ & $42(29.4)$ & & \\
\hline \multirow{2}{*}{$\begin{array}{l}\text { Once a person } \\
\text { has made up } \\
\text { his/her } \\
\text { mind about } \\
\text { committing } \\
\text { suicide no } \\
\text { one can stop } \\
\text { him/her }\end{array}$} & Attempters & $2(6.9)$ & $5(17.2)$ & $15(51.7)$ & $2(6.9)$ & $5(17.2)$ & 13.872 & 0.008 \\
\hline & $\begin{array}{c}\text { Non- } \\
\text { Attempters }\end{array}$ & $4(2.8)$ & $30(21.0)$ & $34(23.8)$ & $48(33.6)$ & $27(18.9)$ & & \\
\hline
\end{tabular}


Global Journal of Intellectual \& Developmental Disabilities

\begin{tabular}{|c|c|c|c|c|c|c|c|c|}
\hline Many suicide & Attempters & $2(6.9)$ & $13(44.8)$ & $6(20.7)$ & $5(17.2)$ & $3(10.3)$ & 3.826 & 0.43 \\
\hline $\begin{array}{c}\text { because of } \\
\text { revenge or to } \\
\text { punish } \\
\text { someone else }\end{array}$ & $\begin{array}{c}\text { Non- } \\
\text { Attempters }\end{array}$ & $27(18.9)$ & $44(30.8)$ & $29(20.3)$ & $31(21.7)$ & $12(8.4)$ & & \\
\hline People who & Attempters & $0(0)$ & $8(27.6)$ & $7(24.1)$ & $9(31.0)$ & $5(17.2)$ & 3.581 & 0.466 \\
\hline $\begin{array}{c}\text { suicide } \\
\text { are usually } \\
\text { mentally ill }\end{array}$ & $\begin{array}{c}\text { Non- } \\
\text { Attempters }\end{array}$ & $14(9.8)$ & $34(23.8)$ & $39(27.3)$ & $35(25.5)$ & $21(14.7)$ & & \\
\hline $\begin{array}{l}\text { It is a human } \\
\text { duty to try }\end{array}$ & Attempters & $15(51.7)$ & $9(31.0)$ & $3(10.3)$ & $2(6.9)$ & $0(0)$ & 2.953 & 0.566 \\
\hline $\begin{array}{l}\text { one from } \\
\text { committing } \\
\text { suicide }\end{array}$ & $\begin{array}{c}\text { Non- } \\
\text { Attempters }\end{array}$ & $53(37.1)$ & $58(40.6)$ & $15(10.5)$ & $12(8.4)$ & $5(3.5)$ & & \\
\hline $\begin{array}{l}\text { When a } \\
\text { person } \\
\text { commits } \\
\text { suicide, it is } \\
\text { something }\end{array}$ & Attempters & $2(6.9)$ & $10(34.5)$ & $12(41.4$ & $5(17.2)$ & $0(0)$ & 3.598 & 0.463 \\
\hline $\begin{array}{l}\text { has } \\
\text { considered } \\
\text { for a long } \\
\text { time }\end{array}$ & $\begin{array}{c}\text { Non- } \\
\text { Attempters }\end{array}$ & $15(10.5)$ & $44(30.8)$ & $44(30.8)$ & $29(20.3)$ & $11(7.7)$ & & \\
\hline $\begin{array}{l}\text { There is a risk } \\
\text { of evoking } \\
\text { suicidal } \\
\text { thoughts in a } \\
\text { person's }\end{array}$ & Attempters & $2(6.9)$ & $19(65.5)$ & $3(10.3)$ & $5(17.2)$ & 0 & 6.931 & 0.14 \\
\hline $\begin{array}{l}\text { mind if you } \\
\text { ask about it }\end{array}$ & $\begin{array}{c}\text { Non- } \\
\text { Attempters }\end{array}$ & $12(8.4$ & $57(39.9$ & $32(22.4)$ & $39(27.3)$ & $3(2.1)$ & & \\
\hline $\begin{array}{l}\text { People who } \\
\text { make suicidal }\end{array}$ & Attempters & $0(0)$ & $7(24.1)$ & $13(44.8)$ & $6(20.7)$ & $3(10.3)$ & 9.544 & $0.049 *$ \\
\hline $\begin{array}{l}\text { threats } \\
\text { seldom } \\
\text { complete } \\
\text { suicide }\end{array}$ & $\begin{array}{c}\text { Non- } \\
\text { Attempters }\end{array}$ & $9(6.3)$ & $40(28.0)$ & $29(20.3)$ & $51(35.7)$ & $14(9.8)$ & & \\
\hline Suicide is a & Attempters & $2(6.9)$ & $9(31.0)$ & $6(20.7)$ & $5(17.2)$ & $7(24.1)$ & 10.327 & $0.035^{*}$ \\
\hline $\begin{array}{l}\text { rather not } \\
\text { talk about }\end{array}$ & $\begin{array}{c}\text { Non- } \\
\text { Attempters }\end{array}$ & $11(7.7)$ & $38(26.6)$ & $30(21.0)$ & $54(37.8)$ & $10(7.0)$ & & \\
\hline Loneliness & Attempters & $4(13.8)$ & $15(51.7$ & $3(10.3)$ & $6(20.7)$ & $1(3.4)$ & 3.087 & 0.543 \\
\hline $\begin{array}{l}\text { reason to take } \\
\text { my life }\end{array}$ & $\begin{array}{c}\text { Non- } \\
\text { Attempters }\end{array}$ & $19(13.3)$ & $56(39.2)$ & $27(18.9)$ & $27(18$. & $14(9.8)$ & & \\
\hline
\end{tabular}


Global Journal of Intellectual \& Developmental Disabilities

\begin{tabular}{|c|c|c|c|c|c|c|c|c|}
\hline \multirow{2}{*}{$\begin{array}{c}\text { Almost } \\
\text { everyone has } \\
\text { at one } \\
\text { time or } \\
\text { another } \\
\text { thought about } \\
\text { suicide }\end{array}$} & Attempters & $3(10.3)$ & $16(55.2)$ & $6(20.7)$ & $2(6.9)$ & $2(6.9)$ & 5.871 & 0.209 \\
\hline & $\begin{array}{c}\text { Non- } \\
\text { Attempters }\end{array}$ & $18(12.6)$ & $56(39.2)$ & $32(22.4)$ & $33(23.1)$ & $4(2.8)$ & & \\
\hline \multirow{2}{*}{$\begin{array}{l}\text { There may } \\
\text { be situations } \\
\text { where } \\
\text { the only } \\
\text { reasonable } \\
\text { resolution is } \\
\text { suicide }\end{array}$} & Attempters & $5(17.2)$ & 11(37.9) & $3(10.3)$ & $7(24.1)$ & $3(10.3)$ & 6.995 & 0.136 \\
\hline & $\begin{array}{c}\text { Non- } \\
\text { Attempters }\end{array}$ & $10(7.0)$ & $35(24.5)$ & $32(22.4)$ & $46(32.2)$ & $20(14.0)$ & & \\
\hline \multirow{2}{*}{$\begin{array}{c}\text { I could say } \\
\text { that I would } \\
\text { take } \\
\text { my life } \\
\text { without } \\
\text { actually } \\
\text { meaning to } \\
\text { do so }\end{array}$} & Attempters & 0 & $7(24.1)$ & $16(55.2)$ & $4(13.8)$ & $2(6.9)$ & 8.46 & 0.076 \\
\hline & $\begin{array}{c}\text { Non- } \\
\text { Attempters }\end{array}$ & $13(9.1)$ & $18(12.6)$ & $62(43.4)$ & $44(30.8)$ & $6(4.2)$ & & \\
\hline \multirow{2}{*}{$\begin{array}{c}\text { Suicide can } \\
\text { sometimes } \\
\text { be a } \\
\text { relief for } \\
\text { those } \\
\text { involved }\end{array}$} & Attempters & $2(6.9)$ & $5(17.2)$ & $7(24.1)$ & $8(27.6)$ & $7(24.1)$ & 6.929 & 0.14 \\
\hline & $\begin{array}{c}\text { Non- } \\
\text { Attempters }\end{array}$ & $16(11.2)$ & $29(20.3)$ & $30(21.0)$ & $56(39.2)$ & $12(8.4)$ & & \\
\hline $\begin{array}{c}\text { Suicides } \\
\text { among young } \\
\text { people are } \\
\text { particularly } \\
\text { puzzling } \\
\text { since } \\
\text { they have } \\
\text { everything to }\end{array}$ & Attempters & $4(13.8)$ & $6(20.7)$ & $13(44.8)$ & $4(13.8)$ & $2(6.9)$ & 6.437 & 0.169 \\
\hline live for & $\begin{array}{c}\text { Non- } \\
\text { Attempters }\end{array}$ & $20(14.0)$ & $42(29.4)$ & $44(30.8)$ & $35(24.5)$ & $2(1.4)$ & & \\
\hline \multirow{2}{*}{$\begin{array}{l}\text { I would } \\
\text { consider the } \\
\text { possibility of } \\
\text { taking my life } \\
\text { if I were to } \\
\text { suffer from } \\
\text { a severe, } \\
\text { incurable, } \\
\text { disease }\end{array}$} & Attempters & $2(6.9)$ & $8(27.6)$ & $8(27.6)$ & $10(34.5)$ & $1(3.40$ & 5.29 & 0.259 \\
\hline & $\begin{array}{c}\text { Non- } \\
\text { Attempters }\end{array}$ & $23(16.1)$ & $25(23.1)$ & $33(23.1)$ & $42(29.4)$ & $20(14.0)$ & & \\
\hline \multirow{2}{*}{$\begin{array}{l}\text { A person } \\
\text { once they } \\
\text { have suicidal } \\
\text { thoughts } \\
\text { will never let } \\
\text { them go }\end{array}$} & Attempters & $6(20.7)$ & 11(37.9) & $4(13.8)$ & $7(24.1)$ & $1(3.4)$ & 1.002 & 0.91 \\
\hline & $\begin{array}{c}\text { Non- } \\
\text { Attempters }\end{array}$ & $25(17.50$ & $50(35.0)$ & $30(21.0)$ & $31(21.7)$ & $7(4.9)$ & & \\
\hline
\end{tabular}


Global Journal of Intellectual \& Developmental Disabilities

\begin{tabular}{|c|c|c|c|c|c|c|c|c|}
\hline \multirow{2}{*}{$\begin{array}{l}\text { Suicide } \\
\text { happens } \\
\text { without } \\
\text { warning }\end{array}$} & Attempters & $2(6.9)$ & $13(44.8)$ & $3(10.3)$ & $9(31.0)$ & $2(6.9)$ & 13.274 & $0.010^{*}$ \\
\hline & $\begin{array}{c}\text { Non- } \\
\text { Attempters }\end{array}$ & $29(20.3)$ & $65(45.5)$ & $30(21.0)$ & $13(9.1)$ & $6(4.2)$ & & \\
\hline \multirow{2}{*}{$\begin{array}{l}\text { Most people } \\
\text { avoid talking } \\
\text { about } \\
\text { suicide }\end{array}$} & Attempters & $7(24.1)$ & $8(27.6)$ & $7(21.1)$ & $3(10.3)$ & $4(13.8)$ & 7.39 & 0.117 \\
\hline & $\begin{array}{c}\text { Non- } \\
\text { Attempters }\end{array}$ & $33(23.1)$ & $73(51.0)$ & $19(13.3)$ & $10(7.0)$ & $8(5.6)$ & & \\
\hline \multirow{2}{*}{$\begin{array}{l}\text { If someone } \\
\text { wants to } \\
\text { commit } \\
\text { suicide, it is } \\
\text { his or her } \\
\text { business } \\
\text { and we } \\
\text { should not } \\
\text { interfere }\end{array}$} & Attempters & $1(3.4)$ & $7(24.1)$ & $5(17.2)$ & $10(34.5)$ & $6(20.7)$ & 3.788 & 0.435 \\
\hline & $\begin{array}{c}\text { Non- } \\
\text { Attempters }\end{array}$ & $15(10.50$ & $43(30.1)$ & $20(14.0)$ & $30(21.0)$ & $35(24.5)$ & & \\
\hline \multirow{2}{*}{$\begin{array}{l}\text { It is mainly } \\
\text { loneliness } \\
\text { that drives } \\
\text { people to } \\
\text { suicide }\end{array}$} & Attempters & $6(20.7)$ & $10(34.5)$ & $4(13.80$ & $5(17.2)$ & $4(13.8)$ & 7.531 & 0.11 \\
\hline & $\begin{array}{c}\text { Non- } \\
\text { Attempters }\end{array}$ & $45(31.5)$ & $47(32.9)$ & $31(21.7)$ & $15(10.5)$ & $5(3.5)$ & & \\
\hline \multirow{2}{*}{$\begin{array}{l}\text { A suicide } \\
\text { attempt is } \\
\text { essentially } \\
\text { a cry for help }\end{array}$} & Attempters & $4(13.8)$ & $15(51.7)$ & $10(34.5)$ & $0(0)$ & $0(0)$ & 8.604 & 0.072 \\
\hline & $\begin{array}{c}\text { Non- } \\
\text { Attempters }\end{array}$ & $33(23.1)$ & $61(42.7)$ & 27(18.9) & $14(9.8$ & $8(5.6)$ & & \\
\hline \multirow{2}{*}{$\begin{array}{l}\text { On the whole, } \\
\text { I do not } \\
\text { understand } \\
\text { how people } \\
\text { can take their } \\
\text { lives }\end{array}$} & Attempters & $3(10.3)$ & $13(44.8)$ & $12(41.4)$ & $1(3.4)$ & $0(0)$ & 5.014 & 0.286 \\
\hline & $\begin{array}{c}\text { Non- } \\
\text { Attempters }\end{array}$ & $18(12.6)$ & $74(51.7)$ & $34(23.8)$ & $10(7.0)$ & $7(4.9)$ & & \\
\hline \multirow{2}{*}{$\begin{array}{c}\text { Usually } \\
\text { relatives have } \\
\text { no idea about } \\
\text { what is going } \\
\text { on when a } \\
\text { person is } \\
\text { thinking of } \\
\text { suicide }\end{array}$} & Attempters & $2(6.9)$ & 15(51.7) & $7(24.1)$ & $3(10.3)$ & $2(6.9)$ & 3.151 & 0.533 \\
\hline & $\begin{array}{c}\text { Non- } \\
\text { Attempters }\end{array}$ & $24(16.80$ & $76(53.1)$ & $27(18.9)$ & $7(4.9)$ & $9(6.3)$ & & \\
\hline $\begin{array}{c}\text { A person } \\
\text { suffering } \\
\text { from a severe, } \\
\text { incurable, } \\
\text { disease }\end{array}$ & Attempters & $2(6.9)$ & $16(55.2)$ & $4(13.8)$ & $3(10.3)$ & $4(13.8)$ & 4.027 & 0.402 \\
\hline \multicolumn{9}{|l|}{$\begin{array}{l}\text { expressing } \\
\text { wishes to die } \\
\text { should }\end{array}$} \\
\hline $\begin{array}{l}\text { get help to } \\
\text { do so }\end{array}$ & $\begin{array}{c}\text { Non- } \\
\text { Attempters }\end{array}$ & $17(11.9)$ & $52(36.4)$ & $21(14.7)$ & $18(12.6)$ & $35(24.5)$ & & \\
\hline
\end{tabular}


Global Journal of Intellectual \& Developmental Disabilities

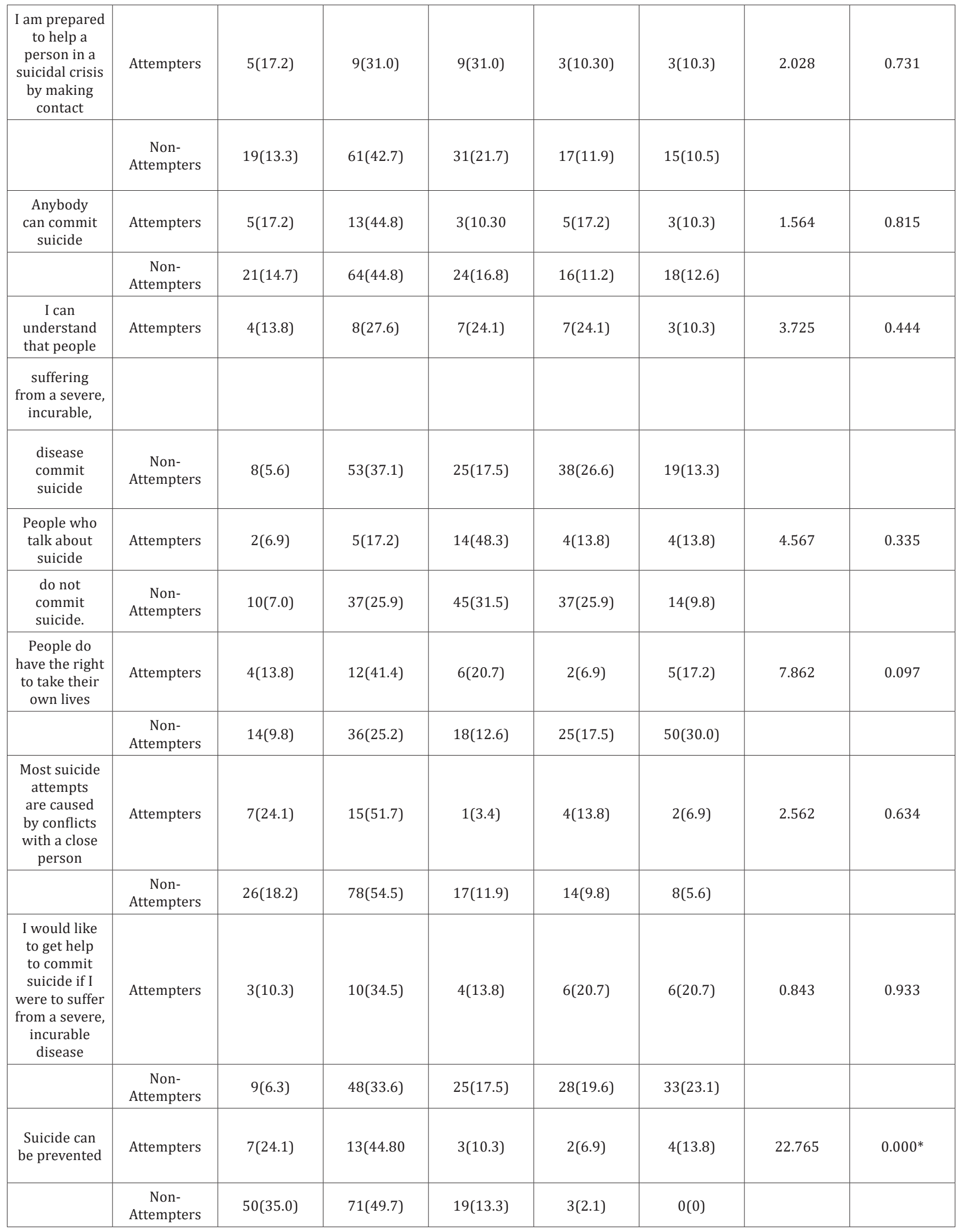




\section{Discussion}

Suicidal thoughts and feelings interfere in the day to-day functioning of an individual and lead to decreased productivity over a period of time. It is possible to recognize those who are vulnerable to suicides. Some of the common warning symptoms include-loss of interest in day to day activities, disturbances in sleep, feeling isolated/dejected/depressed, excessive sadness and crying, communicating about suicidal thoughts, excessive smoking and drinking, feeling non communicative, sudden or gradual change in behavior, etc. These symptoms though gradual in onset, become repetitive, progressive and severe with passage of time.

The present study found that, most of the study subjects were from the age group of 26-35 years, also there is no significant association between their attitudes towards suicide. Majority were married, there is no significant association between married, unmarried and other category towards the suicidal attitudes Majority of the study subjects were females,there is no significant association between attitudes of the male and female study subjects. But contrarily to this other study found significant differences between males and females [17]. This kind of gender specific attitude was also reported in the adult population in other countries $[15,18]$. Many of the study subjects were from Muslim category; also there is no significant association between the attitudes of the study subjects based on the religion. Majority of the respondents who attended and not attended for the suicide, were studied primary education, there is no significant association between them based on the education.

Maximum of the study subjects expressed and agreed that it is always possible to help a person having suicidal thoughts. They also expressed and agreed that suicide can never be justified andsaid that people who commit suicide are usually mentally ill there is no significant association between them also. Similar result found by other researchers also as follows, mental disorders occupy a premier position in the matrix of causation of suicide. Majority of studies note that around $90 \%$ of those who die by suicide have a mental disorder [19].

Countless experts have found that affective disorders are the most important diagnosis related to suicide. In Chennai, 25\% of completed suicides were found to be due to mood disorders. However, the suicide rate increased to $35 \%$ when suicide cases with adjustment disorder with depressed mood were also counted. The crucial and causal role of depression in suicide has limited validity in India. Even those who were depressed, for a short duration and had only mild to moderate symptomatology. The majority of cases committed suicide during their very first episode of depression and more than $60 \%$ of the depressive suicides had only mild to moderate depression [20].

Many of the attempted and non attempted study respondents agreed and strongly said that it is human duty to try to stop someone from committing suicide. Also they expressed, agreed and strongly said that they would consider the possibility of taking their life if they suffer from a sever incurable disease and people who make suicidal threats seldom complete suicide. At the same time our respondents were said that suicide is a subject that one should rather not talk about, it might happen without Similar concept were found and discussed in their study as many beliefs andexplain negative attitude. Chief among these is that suicide is a personal matter that should be left for the individual to decide. Another belief is that suicide cannot be prevented because its major determinants are social and environmental factors such as unemployment over which an individual has relatively little control. However, for the irresistible majority who slot in suicidal behavior, there is most likely an appropriate substitutedeclaration of the precipitating harms. Suicide is often an enduring solution to a provisional problem [21].

\section{Strength and Weakness}

The strength of the present study is the methodological assessment of attitude towards suicide attempts of the general public's. There are limitations to the generalization of the present study results because, the small sample sizes. Second, the study did not examine the effect of the lethality of the present suicide attempt, and during the assessment did not use a psychometric instrument to measurefuture suicide risk. Nevertheless, participants were conveniently selected.Thirdly it is the first study done in the southern state of India; hence the discussion with supportive article was difficult.

\section{Conclusion}

The health care professionals especially the nurses have more opportunity to come across with patient having suicidal ideation or attempted suicide so suicide risk prediction and prevention of most proportion of suicide is their responsibility. Raising wakefulness about suicide avoidance among general population is vital in developing countries like India. Since the causes of suicides are multiple, there is no single solution that can prevent all suicides. The prevention program need to be tailored for different age, sex, cause and setting.Considering that public attitudes are strongly connected to people's social presentations which help to create social reality [22], the nonjudgmental attitude should be implemented into life skills of human being through media by preventive guidelines, stating that there are better solutions to solving problems than suicide. The health care administrators can conduct in-service education programs for various levels of nursing professionals working with suicidal patients. Nurse administrator should see that courses and workshops for nurses are conducted to update their knowledge there by improve the quality of care that provided to the suicidal patients.

\section{Acknowledgement}

Researchers heart fully thank the participants for their valuable contribution. 


\section{References}

1. Gururaj G, Isaac MK, Girish N, Subbakrishna DK. Health behavior surveillance in respect of mental health. Pilot study final report. National Institute of Mental Health and Neuro Sciences, Bangalore, India.

2. WHO (1999) Figures and facts about suicide (No. WHO/MNH/ MBD/99.1). World Health Organisation, Geneva, Switzerland.

3. WHO (2002) Suicide prevention in Europe. World Health Organisation, Geneva, Switzerland.

4. Maris RW (2002) Suicide. Lancet 360(9329): 319-326.

5. Aaron R, Joseph A, Abraham S, Muliyil J, George K, et al. (2004) Suicides in young people in rural southern India. Lancet 363(9415): 1117-1118.

6. Abraham VJ, Abraham S, Jacob KS (2005) Suicide in the elderly in Kaniyambadi block, Tamil Nadu, South India. Int J Geriatr Psychiatry 20(10): 953-955.

7. Joseph A, Abraham S, Muliyil JP, George K, Prasad J, et al. (2003) Evaluation of suicide rates in rural India using verbal autopsies, 1994 9. British Medical Journal 326: 1121-1122.

8. Prasad J, Abraham VJ, Minz S, Abraham S, Joseph A, et al. (2006) Rates and factors associated with suicide in Kaniyambadi Block,Tamil Nadu, South India, 2000-02. Int J Soc Psychiatry 52: 65-71.

9. Tondo L, Baldessarini RJ (2005) Suicidal risk in bipolar disorder Clinical Neuropsychiatry 2(1): 55-65.

10. Kessler RC, Berglund P, Borges G, Nock M, Wang PS (2005) Trends in suicide ideation, plans, gestures, and attempts in the United States, 1990-1992 to 2001-2003. JAMA 293(20): 2487-2495.

11. Farberow NL (1950) Personality patterns of suicidal mental hospital patients. Genet Psychol Monogr 42: 3-79.
12. Farberow NL, Shneidman ES (1955) Attempted, threatened, and completed suicide. Journal of Abnormal and Social Psychology 50(2): 230.

13. Rus VS (1997) Socialna psihologija: teorija, empirija, eksperiment in uporaba II (Social psychology: theory, practise, experiment and use II). Daveand oo, Slovenia, Ljubljana.

14. Nastran Ule M (1997) Temeljisocialnepsihologije (Essentials of social psychology). Znanstveno in publicističnosredišče, Ljubljana, Slovenia.

15. Renberg ES, Jacobsson L (2003) Development of a questionnaire on attitudes towards suicide (atts) and its application in a swedish population. Suicide Life Threat Behav 33(1): 52-64.

16. Mann JJ (2002) A current perspective of suicide and attempted suicide. Ann Intern Med 136(4): 302-311.

17. Kavalidou K (2013) Suicidal thoughts and attitudes towards suicide among medical and psychology students in Greece. Suicidology Online 4: 4-11.

18. Dahlen ER, Canetto SS (2002) The role of gender and suicide precipitant in attitudes toward nonfatal suicidal behavior. Death Stud 26(2): 99116.

19. Vijayakumar L, John S, Pirkis J, Whiteford H (2005) Suicide in developing countries (2): Risk factors. Crisis 26:112-119.

20. Vijayakumar L, Rajkumar S (1999) Are risk factors for suicide universal? A case control study in India. Acta Psychiatr Scand 99: 407-411.

21. Manoranjitham SD, Jayakaran R, Jacob KS (2006) Suicide in India: The need for a national policy.Indian J Psychiatry 48(1): 72.

22. Cernigoj M (2000) Predstavitev in kritikateorije social nihpredstav (The theory of social representations: overview and critique). Psihološkaobzorja 9(1): 23-38.

\section{Your next submission with Juniper Publishers will reach you the below assets}

- Quality Editorial service

- Swift Peer Review

- Reprints availability

- E-prints Service

- Manuscript Podcast for convenient understanding

- Global attainment for your research

- Manuscript accessibility in different formats

( Pdf, E-pub, Full Text, Audio)

- Unceasing customer service

Track the below URL for one-step submission https://juniperpublishers.com/online-submission.php 